\title{
LOW-FREQUENCY PRESSURE FLUCTUATION DAMPER BASED ON HYDROPNEUMATIC SPRING WITH CONSTANT STIFFNESS
}

\author{
Andrzej Figiel, Zygmunt Kudźma, Michae Stosiak \\ Wrockaw University of Science and Technology, Faculty of Technology and Engineering, Wroctaw, Poland \\ e-mail: andrzej.figiel@pwr.edu.pl; michal.stosiak@pwr.edu.pl
}

\begin{abstract}
A wide spectrum of pressure fluctuation frequencies occurs in hydraulic systems. Particularly hazardous and difficult to eliminate are pressure fluctuations in the range up to $50 \mathrm{~Hz}$, resulting in the generation of infrasounds by machines equipped with a hydrostatic drive. The best protection against the harmful effect of ultrasounds is to suppress them at the very source, i.e. to eliminate the causes of the generation of this noise. This paper presents a concept of reducing pressure fluctuation in the range of low excitation frequencies by means of a low-frequency damper of special design. The basis for designing pressure fluctuation dampers effective in reducing pressure fluctuation amplitudes in the range of low frequencies $(<50 \mathrm{~Hz})$, which also function as acoustic filters of the generated infrasounds, is provided. The effectiveness of the low-frequency damper in reducing pressure fluctuation amplitudes has been experimentally tested. The damper was found to be most effective when its eigenfrequency coincided with the excitation frequency to be reduced.
\end{abstract}

Keywords: damper, pressure fluctuation, infrasounds

\section{Introduction}

Pressure fluctuation, on the one hand, is a consequence of the periodically changing rate of flow of the working medium due to cyclicality of operation of pump displacement elements and, on the other hand, it is a result of external excitations in the form of mechanical vibrations acting on the hydraulic system components mounted on various supporting structures (e.g. loader frame). Fluctuations in the output, and so in pressure, in a hydraulic system are undesirable since they can cause many disturbances to normal operation and be a source of excessive noise, see Michałowski and Stolarski (1998). One of the most serious consequences of pressure fluctuations are vibrations of flexible pipes (German et al., 2000; Stosiak, 2011; Czerwinski and Luczko, 2015) and, in some cases, of hydraulic system control elements (Kollek et al., 2010). In order to confirm the coincidence between pressure fluctuations and the emitted noise, Osiński and Kollek (2013) recommend to locate the noise sources by means of a two-microphone probe. In this way, one can obtain a map of noise intensity around the investigated device and indicate the noisiest places which, as a rule, coincide with the areas where the maximum pressure fluctuation amplitude occurs.

A wide pressure fluctuation frequency spectrum, in both the infrasonic (up to $50 \mathrm{~Hz}$ ) range and the audible range (up to $2 \mathrm{kHz}$ ), occurs in hydraulic systems (Ijas, 2007; Kollek et al., 2009; Mikota, 2000; Earnhart and Cunefare, 2012).

The necessity to reduce low-frequency pressure fluctuation on the one hand is dictated by ergonomics and on the other by reduction of low-frequency excitations leading to minimization of the resonant vibration of hydraulic system components such as flexible pipes, valves, controllers and distributors, whose eigenfrequencies are in the low-frequency range. Especially hazardous and difficult to eliminate are pressure fluctuations in the range up to $50 \mathrm{~Hz}$, resulting in the generation of infrasounds by machines equipped with a hydrostatic drive system (Kudźma, 2012). 
The best protection against the harmful effect of ultrasounds is to suppress them at the very source, i.e. to eliminate the causes of the generation of this noise. Various hydropneumatic accumulators are commonly used to reduce pressure fluctuation amplitudes in the low frequency range (Dindorf, 2004; Palczak and Pomowski, 2006; Ijas, 2007; Earnhart and Cunefare, 2012; Kollek et al., 2009; Garbacik et al., 1986). However, the limitation of this solution is that hydropneumatic accumulators are most effective in reducing pressure fluctuation amplitudes when the accumulator resonance frequency coincides with the fluctuation frequency which is to be reduced. It should be noted that the accumulator free vibration frequency to a large extent depends on the magnitude of pressure in the hydraulic system incorporating this component. This means that hydropneumatic accumulators act selectively and so as dampers they are narrow-band filters, and their actual effectiveness in reducing pressure fluctuation is limited to practically a single excitation frequency equal to the hydropneumatic accumulator resonance frequency changing with the load of the drive system which incorporates the accumulator. Damping effectiveness tends to rapidly decrease already at slight deviations from the resonance frequency (Ortwig et al., 1999; Kudźma, 2012; Garbacik et al., 1986). The minimization of pressure fluctuations in hydraulic systems through the use of various dampers has been investigated by, among others, Dindorf (2004), Ijas (2007), Kudźma (2012), Kudźma and Kudźma (2015), Kollek et al. (2009), Earnhart and Cunefare (2012), Garbacik et al. (1986), Mikota (2000), Ortwig et al. (1999), Singh (2005), Skaistis (1988). Most of the above authors found that passive chamber dampers of the bypass, branch and Helmholtz resonator type were usable at excitation frequencies above $150 \mathrm{~Hz}$. This limitation is due to the fact that for lower excitation frequencies the above dampers assume overall geometrical dimensions which eliminate them from the use in real hydrostatic drive systems of machines. A Helmholtz resonator with flexible lining of its walls, effective in reducing amplitudes of low (below $50 \mathrm{~Hz}$ ) pressure fluctuation frequencies, but at the maximum pressure in the system limited to a few MPa, was presented by Earnhart and Cunefare (2012). The application of active damping consisting in pressure fluctuation generation by means of complex electrohydraulic systems in counterphase to the pressure fluctuation which is to be reduced, was presented by Changbin and Zongxia (2014), Pan (2013). By properly adjusting the phase and amplitude of the additional fluctuation it is possible to effectively reduce the fluctuation generated by the pump in the whole frequency range. Because of its complicated structure, the active damping of pressure fluctuation has not gone beyond laboratory tests.

This paper presents a concept for reducing pressure fluctuation in the range of low excitation frequencies through a special design of the low-frequency damper effective in the whole range of loads.

\section{Low-frequency pressure fluctuation damper}

In order to reduce low-frequency pressure fluctuation amplitudes, a damper whose design (laboratory version) is shown in Fig. 1 has been used. In comparison with the patent damper, this design was modified by introducing an additional piston rod to enable a simple change of the total vibrating system weight and measurements of piston displacements during damper operation. The damper is a kind of bypass in the hydraulic system, performing function of a reactive

filter and a filter absorbing changes in energy resulting from output and pressure fluctuations. It reduces low-frequency output fluctuations. Thanks to the use of two hydropneumatic accumulators differently charged with gas, the damper effectively performs its function in a wide range of operational system pressures.

The low-frequency fluctuation damper shown in Fig. 1 operates as described below. After the cylinder is filled with the hydraulic oil, hydropneumatic accumulators 1 and 2 mounted in top cover 9 are precharged with nitrogen to pressure $p_{g 02}>p_{g 01}$. The damper is connected 


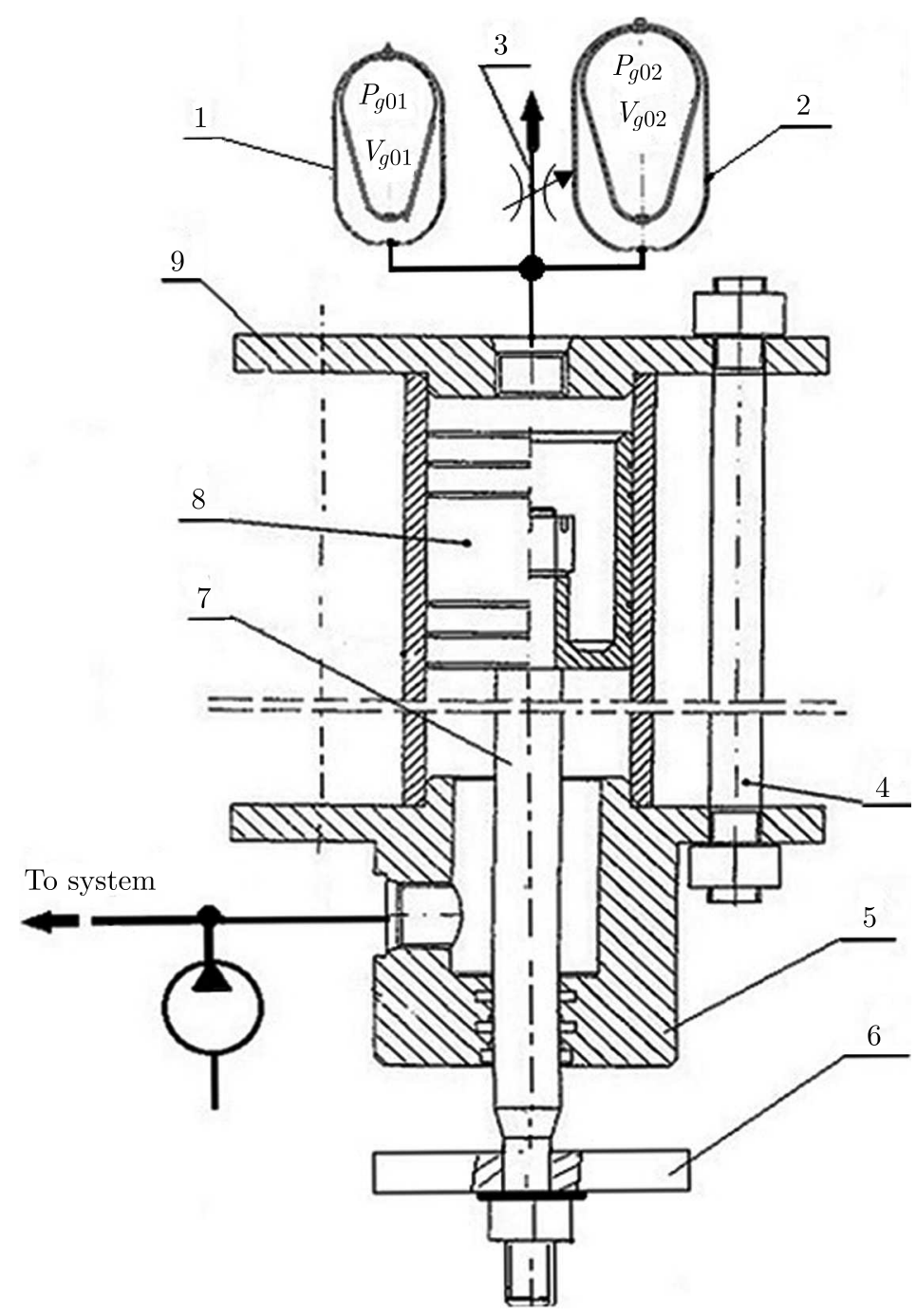

Fig. 1. Active low-frequency pressure fluctuation damper in its laboratory version: 1 - hydraulic accumulator, 2 - hydraulic accumulator, 3 - vent and cut-off valve, 4 - pin, 5 - bottom cover, 6 - loading disk, 7 - piston rod, 8 - piston, 9 - top cover

in parallel via a threaded hole in bottom cover 5 to the pump pressure conduit. The principle of operation of the damper comes down to taking over output fluctuation (generating pressure fluctuation) excitations. The excitations are taken over by the system: the movable piston with the piston rod - a hydropneumatic spring. The hydropneumatic spring is made up of at least two hydraulic accumulators differing in their initial gas volumes and precharge pressures. Owing to this, the hydropneumatic spring stiffness is approximately constant in the whole range of pressures generated by the driver element of the hydraulic system and, consequently, the low-frequency damper effectiveness is constant in the whole range of loads. Because of the patent application being processed, the low-frequency damper is only generally described here.

\section{Mathematical model}

\subsection{First stage in damper operation}

The operation of the low-frequency damper can be divided into two stages. The first stage covers the period from starting the system to the instant when the average pressure $p_{a v}$ is lower 
than the precharge pressure $p_{g 02}$ in accumulator 2 (Fig. 1) - only one accumulator operates. The second stage begins when the average system pressure $p_{a v}>p_{g 02}$ - both accumulators operate.

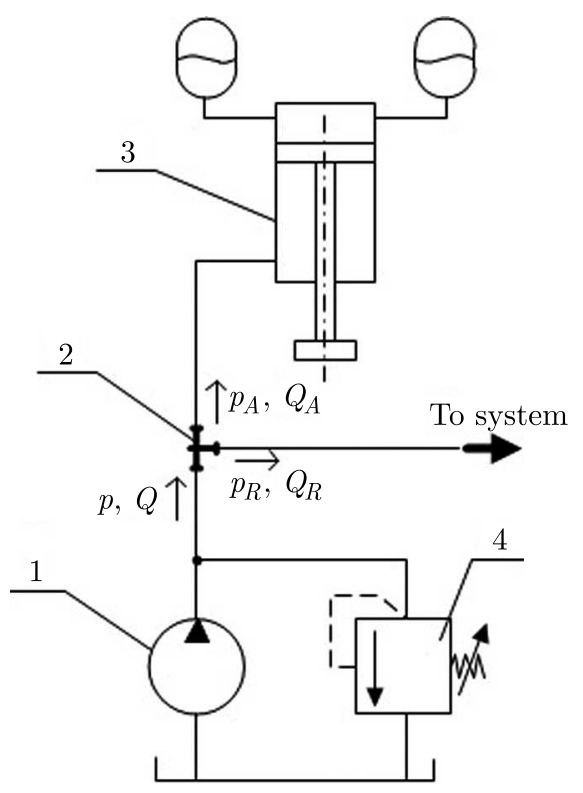

Fig. 2. Way of installing the low-frequency damper in the system: 1 - displacement pump, 2 - tee TT (feed node), 3 - low-frequency damper, 4 - safety valve

The particular symbols in Fig. 2 stand for: $Q$ - the rate of pump delivery, $Q_{A}$ - rate of flow to the low-frequency damper, $Q_{R}$ - rate of flow to the pipeline feeding the system, and $p \cong p_{A} \cong p_{R}$ - pressure deviations in tee (tee arms).

Analysis of the hydraulic system with the low-frequency damper begins with calculation of the operational impedance $Z_{T}(s)$ in the feed node $T_{T}$

$$
Z_{T}(s)=\frac{p_{A}(s)}{Q_{A}(s)}
$$

where $p_{A}(s)$ and $Q_{A}(s)$ are the Laplace transforms of the pressure $p_{A}$ and flow rate $Q_{A}, s$ is the Laplace variable.

On the basis of the calculated impedance one selects such its value which would ensure minimal changes in $Q_{R}$ and in pressure $p_{R}$. The parameters are a result of the pulsatory flow rate in the infrasonic range.

Moreover, the following simplifying assumptions are made:

- the system operates at thermal equilibrium with its surroundings;

- compressibility of the oil and elasticity of the conduits are neglected as being small in comparison with elasticity of the gas in the accumulator, also leakage losses are neglected;

- the safety valve remains closed over the whole load range;

- at low $Q_{A}$ values, the flow in the low-frequency damper and the hydraulic accumulators connections is laminar.

For the feed node $T_{T}$, on the above assumptions, the equation of continuity for a nonuniform flow can be expressed through constant flow components $Q_{A S}, Q_{R S}, Q_{S}$ and variable flow components $Q_{A}, Q_{R}$ and $Q$

$$
Q_{S}+Q=Q_{R S}+Q_{R}+Q_{A S}+Q_{A}
$$

For the system shown in Fig. 2, one can assume that the whole flow generated by the pump goes via connection $T$ to the system: damper-pipeline feeding the system. Thus the equation of 
flow continuity assumes form (3.2): $Q+Q_{S}=Q_{R S}+Q_{R}+Q_{A S}+Q_{A}$. Moreover, the whole (mean) constant flow $\left(Q_{S}\right)$ in steady operating conditions goes to the pipeline which feeds the system: $Q_{S}=Q_{R S}$. Thus the value of the constant component going to the damper is $Q_{A S}=0$. Therefore, equation (3.2) can be written as

$$
Q=Q_{A}+Q_{R}
$$

The tee transmittances can be presented in the form of the matrix equation, see Kudźma (2012), Earnhart and Cunefare (2012)

$$
\left[\begin{array}{l}
p \\
Q
\end{array}\right]=\left[\begin{array}{cc}
1 & 0 \\
Z_{T}^{-1} & 1
\end{array}\right]\left[\begin{array}{c}
p_{R} \\
Q_{R}
\end{array}\right]
$$

where $Z_{T}=p_{A} / Q_{A}$ is the impedance of the damping system.

The expansion of equation (3.4) yields

$$
Q_{R}=Q-\frac{p_{R}}{Z_{T}}
$$

The mathematical model of the low-frequency damper is used to determine the structural parameters of the latter ensuring the maximum elimination of system output fluctuations with a given frequency, i.e. $Q_{R}(t)=\min$.

The flow caused by compressibility of the gas in the accumulator is defined by the equation (Dindorf, 2004)

$$
Q_{A}=C_{A} \frac{d p_{g}}{d t}
$$

where $C_{A}$ is the capacitance of the hydraulic accumulator, $p_{g}$ - pressure of the gas in the accumulator.

The capacitance of the hydraulic accumulator and its dynamic properties depend on the mass of the partition (membrane, etc.), compressibility of the gas and the inertia and resistance of the flow of the oil. For a constant entropy $S=$ const, the accumulator capacitance can be written as (Dindorf, 2004; Kudźma, 2012)

$$
C_{A}=\left(\frac{\partial V_{g}}{\partial p_{g}}\right)_{S}
$$

where $V_{g}$ is the volume of the gas in the accumulator.

Taking into account:

- (active) damper resistance $R_{0 T}$ which includes the effect of fluid resistance and piston motion resistance,

- piston hydraulic inductance $L_{T}$ (passive resistance) which includes the effect of inertia of the fluid and moving piston components, one can determine the pressure drop (Palczak and Pomowski, 2006)

$$
p_{A}-p_{g}=L_{T} \frac{d Q_{A}}{d t}+R_{0 T} Q_{A}
$$

From the assumption that hydraulic resistances, expressed by the last term of equation (3.8), have a quasi-stationary character it follows that the fluid friction loss coefficient for the steady flow and for the unsteady flow in the conduit at the same fluid flow velocity has an identical value. Consequently, considering the low flow rate fluctuation frequencies and the relatively large radius of the conduit, the effect of fluid motion nonstationarity in the conduit on frictional resistances is neglected. 
Having differentiated both sides of expression (3.8), one gets

$$
\frac{d p_{A}}{d t}-\frac{d p_{g}}{d t}=L_{T} \frac{d^{2} Q_{A}}{d t^{2}}+R_{0 T} \frac{d Q_{A}}{d t}
$$

Using equation (3.6) and rearranging (3.9), one gets

$$
C_{A} \frac{d p_{A}}{d t}=C_{A} L_{T} \frac{d^{2} Q_{A}}{d t^{2}}+C_{A} R_{0 T} \frac{d Q_{A}}{d t}+Q_{A}
$$

Having applied the Laplace transformation to equation (3.10) at zero initial conditions, one gets the low-frequency damper operational transmittance $G_{T}(s)$

$$
G_{T}(s)=\frac{Q_{A}(s)}{p_{A}(s)}=\frac{C_{A} s}{C_{A} L_{T} s^{2}+C_{A} R_{0 T} s+1}
$$

Assuming the damper eigenfrequency

$$
\omega_{0 T}=\sqrt{\frac{1}{C_{A} L_{T}}}
$$

and the damping number

$$
\xi_{T}=\frac{1}{2 \omega_{0 T}} C_{A} R_{0 T}
$$

one gets

$$
G_{T}(s)=\frac{C_{A} s}{\frac{1}{\omega_{0 T}^{2}} s^{2}+2 \xi_{T} \omega_{0 T} s+1}
$$

The amplitude-frequency characteristics of the low-frequency damper are determined on the basis of its initial impedance $Z_{T}(s)$ in spectral form $\left|Z_{T}(\omega)\right|$. Therefore, $s=\mathrm{j} \omega$ is substituted into the transition function $G_{T}(s)$ (3.11) and inverse of the latter is specified, whereby one gets

$$
Z_{T}(\omega)=\frac{\left(p_{A}(\omega)\right.}{Q_{A}(\omega)}=\frac{1}{C_{A} \omega_{0 T}}\left[2 \xi_{T} \omega_{0 T}^{2}+\mathrm{j}\left(\frac{\omega}{\omega_{0 T}}-\frac{\omega_{0 T}}{\omega}\right)\right]
$$

$\mathrm{j}$ is the imaginary unit.

Assuming constant coefficients related to the low-frequency damper design

$$
\Phi=\frac{1}{C_{A} \omega_{0 T}} \quad \Psi_{T}=2 \xi_{T} \omega_{0 T}^{2}
$$

from formula (3.15), one calculates the impedance modulus

$$
\left|Z_{T}(\omega)\right|=\Phi \sqrt{\Psi_{T}^{2}+\left(\frac{\omega}{\omega_{0 T}}-\frac{\omega_{0 T}}{\omega}\right)^{2}}
$$

In order to generalize the above analysis, relation (3.17) is presented in the dimensionless form

$$
\left|Z_{T B}\right|=\frac{\left|Z_{T}\right|}{\Phi}=\sqrt{\Psi_{T}^{2}+\left(\frac{\omega}{\omega_{0 T}}-\frac{\omega_{0 T}}{\omega}\right)^{2}}
$$

In order to determine the low-frequency damper impedance minimum corresponding to the maximum damping of pressure fluctuations in the connection node, and to relate this value to the 
damper structural parameters, one should define such parameters as: hydraulic inductance $L_{T}$, capacitance $C_{A}$ and damper connection (tee+pipe) resistance $R_{0 T}$.

The hydraulic inductance $L_{T}$ is defined by the expression (Backé and Murrenhoff, 1994)

$$
L_{T}=\frac{M_{z r}}{A_{A}^{2}}
$$

where $M_{z r}$ is the mass reduced to the damper piston face area, $A_{A}$ - active damper piston face area.

The reduced mass $M_{z r}$ of the vibrating system is the sum of the piston mass, loading disk mass, mass of the fluid in the damper cylinder and the reduced-to-the-piston-face-surface mass of the fluid in the connection.

In accordance with the assumptions, the resistance of the damper is calculated from the Hagen-Poiseuelle relation (Palczak and Pomowski, 2006)

$$
R_{0}=\frac{8 \pi \mu L_{c}}{F_{p}^{2}}
$$

where $\mu$ is the dynamic viscosity of the working medium, $F_{p}$ - cross sectional area of the connection, $L_{c}$ - total length of the connection.

Each accumulator has its specific capacitance $C_{A}$. By analogy to pneumomechanics, the flexibility of the hydropneumatic spring is $C_{S}=C_{A} / A_{A}^{2}$. The spring flexibility, in turn, is the inverse of its stiffness, i.e. stiffness $K_{S}$ of the hydropneumatic spring according to Dindorf (1998) is

$$
K_{S}=\frac{1}{C_{S}}=\frac{A_{A}^{2}}{C_{A}}
$$

The stiffness of the hydropneumatic spring should be related to the damper structural parameters $A_{A}$, pressure $p_{g 01}$ and the initial gas volume $V_{g 01}$. Changes in the gas pressure and volume in the hydropneumatic accumulator in quick-variable processes are interrelated by the polytropic equation (Backé and Murrenhoff, 1994)

$$
p_{g 01} V_{g 01}^{n}=p_{1 g} V_{1 g}^{n}
$$

where $p_{g 01}$ is the initial gas pressure, $V_{g 01}$ - initial gas volume, $p_{1 g}$ - ultimate gas pressure, $V_{1 g}$ - ultimate gas volume, $n$ - polytropic exponent.

The change in the gas volume $\left(\Delta V_{g}\right)$ is interrelated with the piston displacement $x$ through the expresion

$$
\Delta V_{g}=A_{A} x
$$

Hence, one can write

$$
V_{1 g}=V_{g 01}-A_{A} x
$$

On the basis of equations (3.22) and (3.24), one can determine the ultimate pressure $p_{1 g}$ depending on the initial pressure $p_{g 01}$, initial volume $V_{g 01}$ and piston displacement $x$

$$
p_{1 g}=\frac{p_{g 01}}{\left(1-\frac{A_{A}}{V_{g 01}} x\right)^{n}}
$$

Multiplying equation (3.25) by the active piston surface $A_{A}$, one gets the relation for the hydropneumatic spring force $P_{s h}$ as a function of the displacement $x$

$$
P_{s h}=\frac{A_{A} p_{g 01}}{\left(1-\frac{A_{A}}{V_{g 01}} x\right)^{n}}
$$


Having linearized equation (3.26), using the expansion into a Maclaurin series and taking the first terms into account, one gets

$$
\Delta P_{s h}=\frac{n A_{A}^{2} p_{g 01}}{V_{g 01}} \Delta x+A_{A} p_{g 01}
$$

where $\Delta P_{s h}$ is an increase in the hydropneumatic spring force, $\Delta x$ - an increase in the piston displacement. The value of the error due to the Lagrange remainder in the Maclaurin formula depends on the $A_{A} / V_{g 01}$ ratio and it approaches its maximum when approaching the value of the inverse to the $A_{A} / V_{g 01}$ ratio because

$$
\frac{p_{g 01}}{\left(1-\frac{A_{A}}{V_{g 01}} x\right)^{n}}=p_{g 01}+\frac{A_{A} n p_{g 01}}{V_{g 01}} x+\frac{A_{A}^{2} n(n+1) p_{g 01}}{2 V_{g 01}^{2}\left(1-\frac{A_{A}}{V_{g 01}} c\right)^{n+3}} x^{2}
$$

hence

$$
\left|\frac{A_{A}^{2} n(n+1) p_{g 01}}{2 V_{g 01}^{2}\left(1-\frac{A_{A}}{V_{g 01}} c\right)^{n+3}}\right| x^{2} \leqslant M x^{2} \quad\left|\frac{A_{A}^{2} n(n+1) p_{g 01}}{2 V_{g 01}^{2}\left(1-\frac{A_{A}}{V_{g 01}} c\right)^{n+3}}\right| \leqslant M
$$

where $c \in(0, x)$.

Using the system stiffness defining relation

$$
K_{s} \stackrel{\text { def }}{=} \frac{\partial P_{s h}}{\partial \Delta x}
$$

one gets the final form of the expression describing the stiffness of the hydropneumatic spring

$$
K_{s}=\frac{n A_{A}^{2} p_{g 01}}{V_{g 01}}
$$

Returning to equation (3.21) and using relation (3.31), after transformations, one gets

$$
\text { (3.32) } C_{A}=\frac{V_{g 01}}{n p_{g 01}}
$$

Equation (3.32) describes the capacitance of the hydroaccumulator in the neighbourhood of the point $x=0$ and for slight changes in the location of this point.

\subsection{Second stage in damper operation}

When the drive unit load increases, the average operating hydrostatic system pressure and the gas pressure in the accumulator change from $p_{01 g}$ to $p_{k g}$. Since the accumulator fills with the oil slowly, one can assume that compression of the gas is an isothermal process in accordance with the equation

$$
p_{g 01} V_{g 01}=p_{k g} V_{k g}
$$

and so as the load increases, motion of the damper piston takes place at the average pressure $p_{k g}$, and the volume of the gas in the accumulator amounts to

$$
V_{k g}=\frac{p_{g 01} V_{g 01}}{p_{k g}}
$$

Substituting $p_{k g}$ for $p_{01 g}$ and $V_{k g}$ for $V_{g 01}$ (a new balance point) in equation (3.31), one gets

$$
K_{s 1}=\frac{n A_{A}^{2} p_{k g}}{p_{g 01} V_{g 01}}
$$


Relation (3.35) describes the stiffness of the hydropneumatic spring of the active damper operating at the average pressure $p_{k}$ in the system. Returning to equation (3.21), in new conditions of the equilibrium, one gets an expression for the accumulator capacitance $C_{A 1}$

$$
C_{A 1}=\frac{p_{g 01} V_{g 01}}{n p_{k g}^{2}}
$$

The change in the accumulator capacitance as a result of an increase in the average pressure from $p_{01 g}$ to $p_{k g}$, and so the change in the accumulator eigenfrequency $\omega_{O T}$ and in damping effectiveness, amounts to

$$
\frac{C_{A 1}}{C_{A}}=\left(\frac{p_{g 01}}{p_{k g}}\right)^{2}
$$

In order to reduce the effect of variation in the average system pressure on the accumulator eigenfrequency at the instant when a certain pressure $p_{k g}$ is reached, the volume of the other accumulator whose precharge pressure amounts to $p_{k g}$ is added. This is the essence of the proposed low-frequency damper solution. In order to prove this thesis, experiments consisting in determining the capacitance for a single accumulator and, starting from a certain pressure value, for a system of two accumulators connected in parallel, have been carried out. A diagram of the measuring system for determining the capacitance of the hydraulic accumulators is shown in Fig. 3.

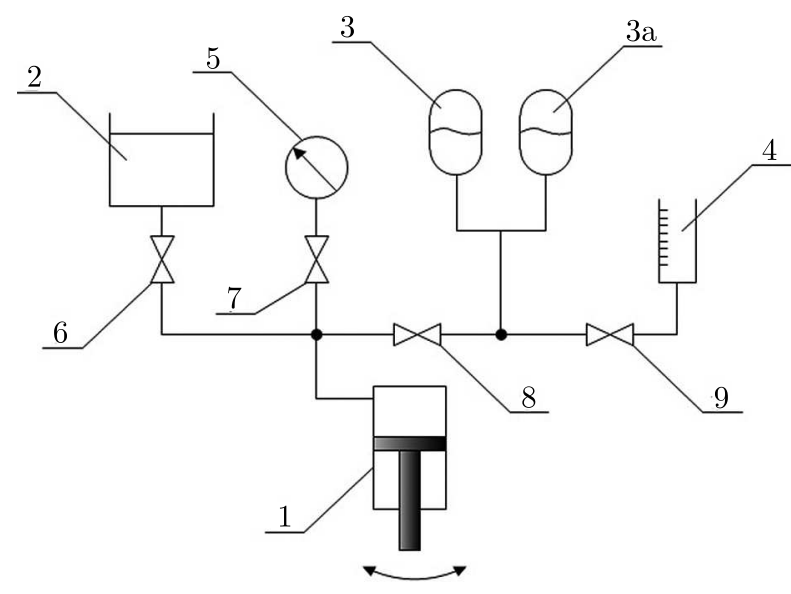

Fig. 3. Diagram of the system for determining the hydropneumatic accumulator capacitance in stationary conditions: 1 - hand-operated pump, 2 - priming tank, 3, 3a - tested hydroaccumulators, 4 - calibrating tank, 6, 7, 8, 9 - cut-off valves, initial accumulator operating conditions $3: V_{0}=0.5 \mathrm{dm}^{3}$, $p_{0}=2 \mathrm{MPa}$; for accumulator $3 \mathrm{a}: V_{0}=0.7 \mathrm{dm}^{3}, p_{0}=8 \mathrm{MPa}$

Using defining relation (3.7) and replacing the partial derivatives with increments, one can experimentally determine the capacitance of the hydropneumatic accumulator via volume changes $\Delta V_{g}$ caused by pressure increment. This is shown in Fig. 3. In order to obtain the required pressure value, hand-operated pump 1 (pressure gauge calibration press) has been used while the volume change $\Delta V_{g}$ was determined using graduated vessel 4 and properly set the cut-off valves (Fig. 4).

Relation $\Delta V_{g}=f\left(p_{g}\right)$ in the pressure range of 2-16 MPa for the two hydropneumatic accumulators was linearized with a secant, and a constant capacitance value $C_{A}=0.56 \cdot 10^{-10} \mathrm{~m}^{5} / \mathrm{N}$ was obtained. 


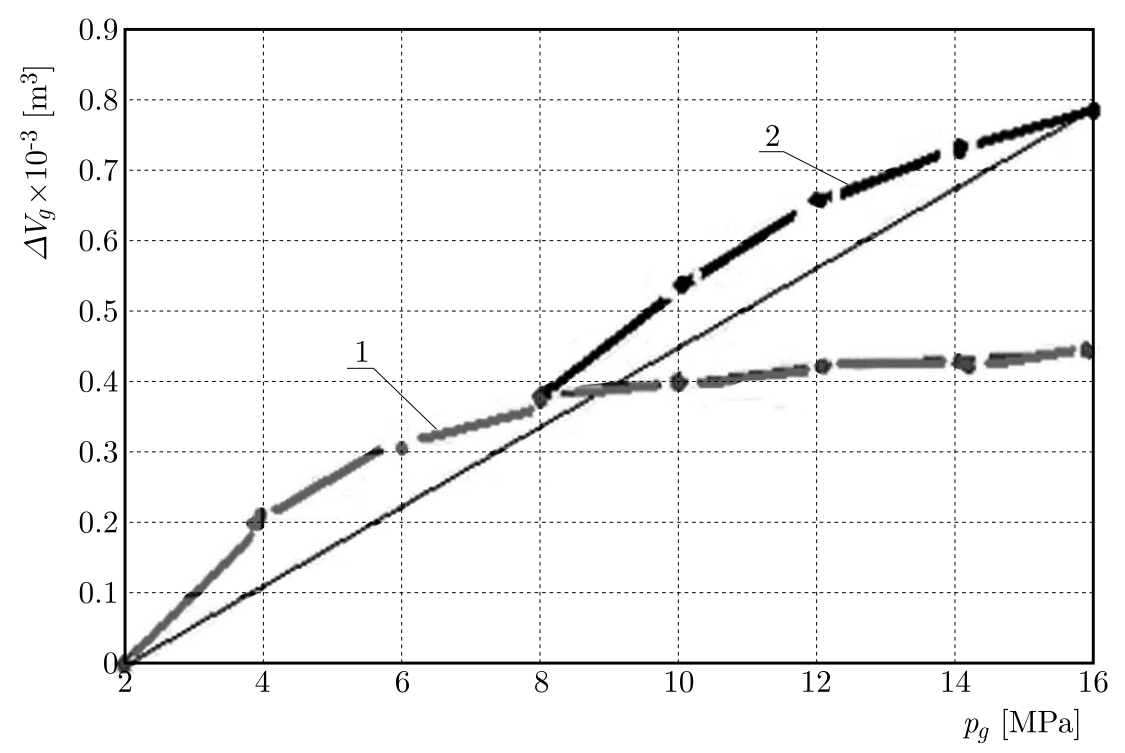

Fig. 4. Change $\Delta V_{g}$ in gas volume in the hydraulic accumulators connected in parallel versus pressure; one accumulator I, $p_{0 g 1}=2 \mathrm{MPa}, V_{0 g 1}=0.5 \mathrm{dm}^{3}$, two accumulators connected in parallel I+II, $p_{0 g 2}=8 \mathrm{MPa}, V_{0 g 2}=0.7 \mathrm{dm}^{3}$

\section{Solution of the mathematical low-frequency damper model}

The solution of the mathematical active damper model, based on relation (3.18), is presented graphically using Mathematica 5.1 and Origin v. 7.5 Pro software. Before solving the model, it was necessary to determine the particular coefficients and their variation ranges, which was done analytically or experimentally. The values of some of the major coefficients are: resultant reduced mass $M_{z r}=2.87 \mathrm{~kg}$, inductance $L_{T}=0.65 \cdot 10^{6} \mathrm{~kg} / \mathrm{m}^{4}$, connection resistance $R_{0 T}=$ $(1.5-50) \cdot 10^{8} \mathrm{Ns} / \mathrm{m}^{5}$, range of dynamic viscosity $\mu=(30-1000) \cdot 10^{-3} \mathrm{Ns} / \mathrm{m}^{2}$, total length $L_{c}=0.86 \mathrm{~m}$, capacitance $C_{A}=0.56 \cdot 10^{-10} \mathrm{~m}^{5} / \mathrm{N}$, eigenfrequency according to formula $(3.12)$ $\omega_{0 T}=158 \mathrm{~s}^{-1}$ (corresponds to eigenfrequency $f_{0 T}=25 \mathrm{~Hz}$ and so to the rotational speed $n_{p}=1500 \mathrm{rpm}$ of the motor driving the displacement pumps in the experiments) and damping number $\xi_{T}=2.9 \cdot 10^{-5} \mathrm{~s}^{2}$.

Figure $5 \mathrm{a}$ shows the effect of oil viscosity on the impedance modulus of the active damper in dimensionless coordinates (other parameters remain unchanged). Figure 5b illustrates the damper impedance modulus-dimensionless frequency relationship for viscosity $\mu=30 \cdot 10^{-3} \mathrm{Ns} / \mathrm{m}^{2}$.
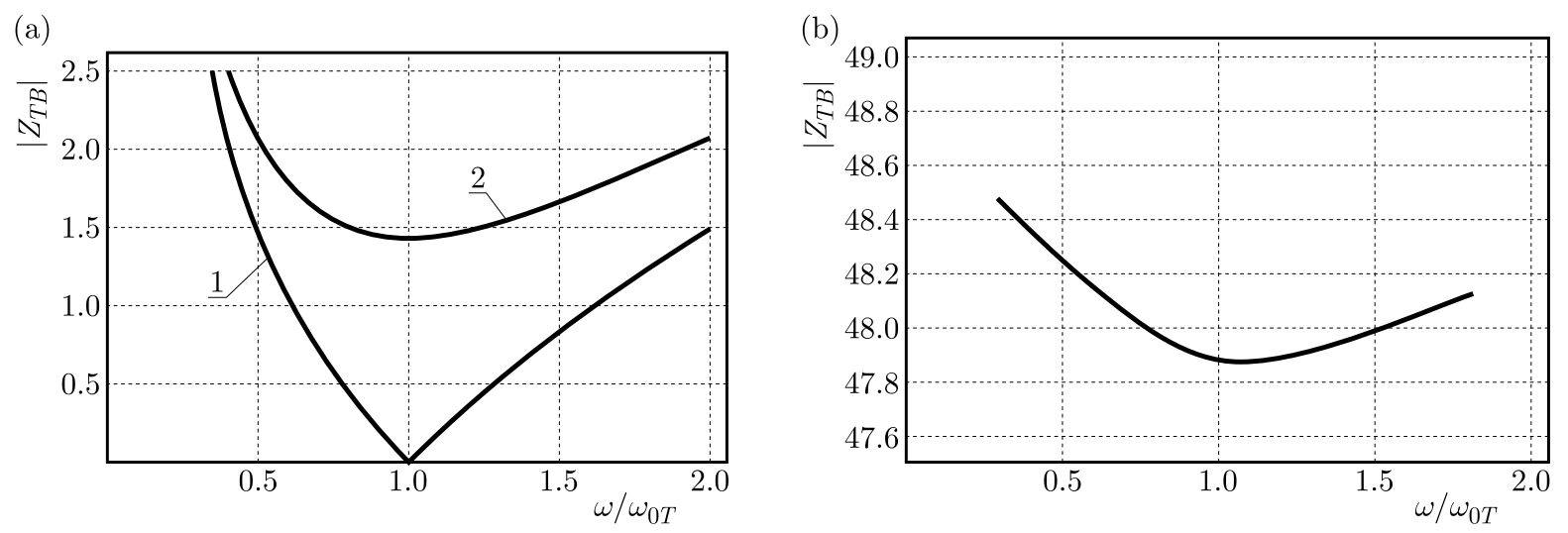

Fig. 5. (a) Low-frequency impedance modulus in dimensionless coordinates: 1 - oil viscosity $\mu=0$;

2 - oil viscosity $\mu=30 \cdot 10^{-3} \mathrm{Ns} / \mathrm{m}^{2}$; low-frequency damper impedance modulus in dimensionless coordinates for oil viscosity $\mu=200 \cdot 10^{-3} \mathrm{Ns} / \mathrm{m}^{2}$ 


\section{Experimental verification}

Hydraulic pressure fluctuation tests involving the low-frequency damper have been carried using a test setup (Fig. 6) designed by the authors. The setup made it possible to determine amplitude-frequency characteristics of the investigated damper. A single-stage VCD (VoiceCoilDrive) Parker-Hannifin D1FPE01MC9NB00 proportional distributor was used as the pressure fluctuation inducing element. This means that, as opposed to conventional proportional distributors, it is not an electromagnet core, but a moving coil which acts on the spool. Consequently, the dynamic performance of the valve is significantly better owing to a substantial reduction in the moving mass (Kolvenbach and Krips, 2004). The distributor specifications were as follows: hysteresis $<0.05 \%$ and the cut-off frequency at $5 \%$ of the command signal $-350 \mathrm{~Hz}$ at the amplitude damping by $3 \mathrm{~dB}$ (Kudźma et al., 2014). Dedicated software called HydroSter was developed to control the proportional valve in the test setup. A sinusoidal command signal with the set amplitude and frequency, generated by the HydroSter software, fed to the distributor coil would induce pressure fluctuations with a specified amplitude and frequency. The structure and operation of the HydroSter software are described in more detail in Kudźma (2012).

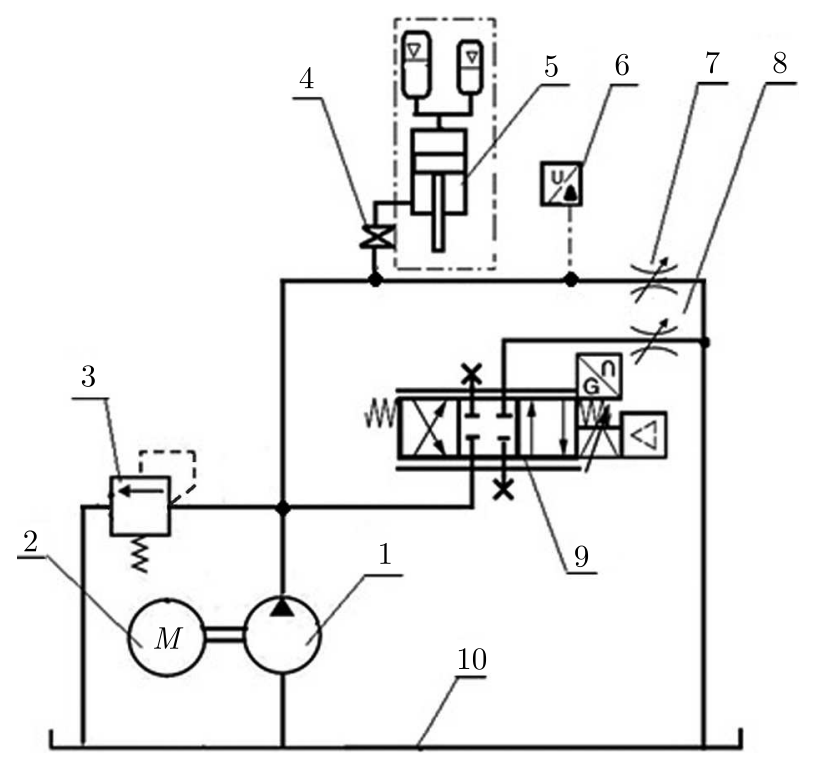

Fig. 6. Hydraulic diagram for determination pf frequency characteristics of a low-frequency damper: 1 - displacement pump, 2 - electric motor, 3 - safety valve, 4 - cut-off valve, 5 - low-frequency damper, 6 - pressure transducer, 7,8 - throttle valve, 9 - proportional distributor, 10 - tank

The experimental verification consisted in recording pressure fluctuations over time while generating pressure fluctuations with the set frequency and amplitude, with the low-frequency damper installed and without the damper, in identical operating conditions. In the system shown in Fig. 6, the sources of excitations in the form of output and pressure fluctuations were: working displacement pump 1 (with a constant delivery and a fixed driving shaft speed) and proportional distributor 9 to whose coils a time-varying command signal with the set amplitude, constant value and frequency was fed. The presence of the damper in the system was determined by opening of cut-off valve 4 (Fig. 6). In the laboratory version of the damper, a displacement transducer was used to measure the displacement of the damper piston. Sample results of pressure fluctuation measurements are presented in Figs. 7-9.

Then the effectiveness of the damper was experimentally verified by determining the amplitude-frequency characteristic for the input pressure fluctuation amplitude $\Delta p$ and the outputpiston displacement $\Delta x$. As a part of the investigations, the resonance frequency corre- 


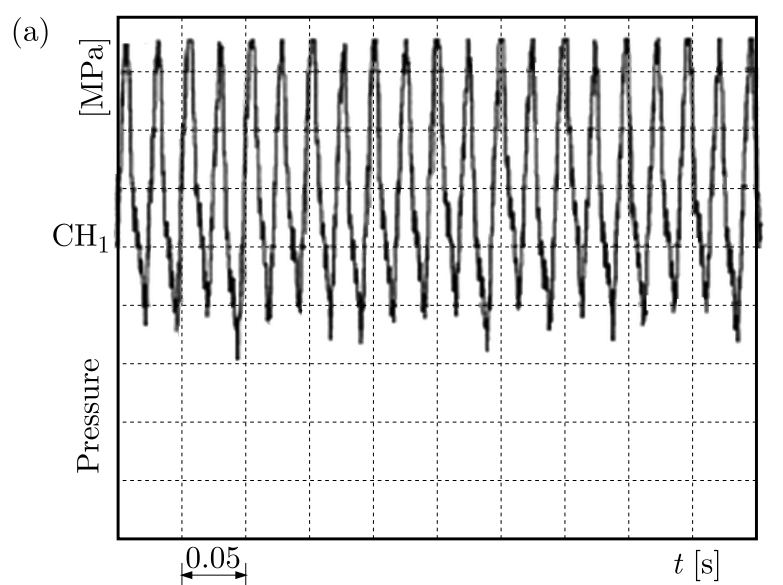

(b)

Fig. 7. Pressure fluctuations in the hydraulic system. Frequency of the pressure fluctuation excitation with a harmonic exciter $f_{w}=25 \mathrm{~Hz}$. Forcing pressure $p_{t}=12 \mathrm{MPa}$ : (a) system without the damper,

(b) system with the damper (the scales in the two figures are identical)

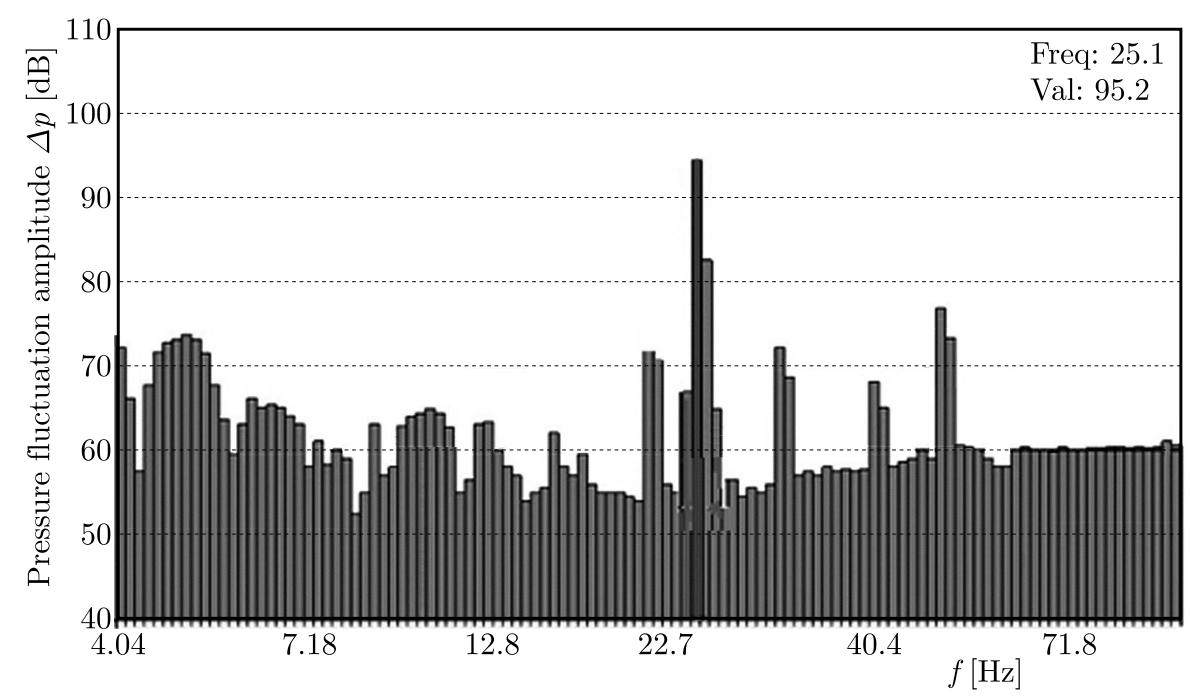

Fig. 8. Narrow-band analysis of pressure fluctuation in the hydraulic system without the low-frequency damper. Frequency of the pressure fluctuation excitation with a harmonic exciter $f_{w}=25 \mathrm{~Hz}$. Forcing pressure $p_{t}=12 \mathrm{MPa}$

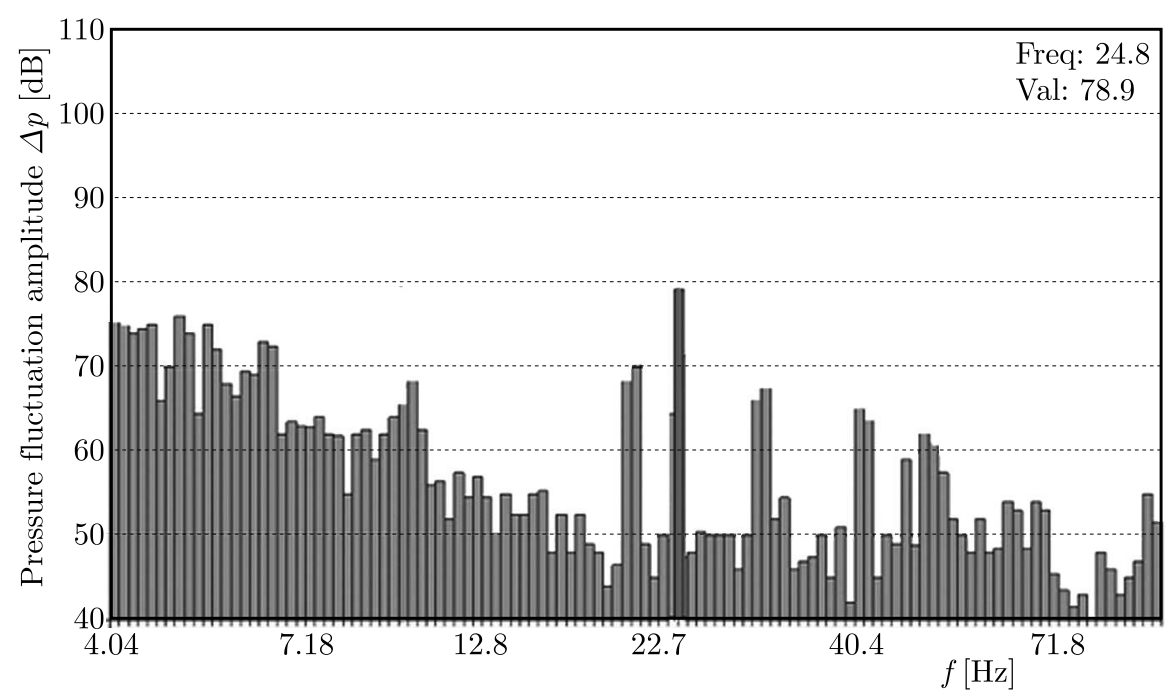

Fig. 9. Narrow-band analysis of pressure fluctuation in the hydraulic system with the low-frequency damper. Frequency of the pressure fluctuation excitation with a harmonic exciter $f_{w}=25 \mathrm{~Hz}$. Forcing pressure $p_{t}=12 \mathrm{MPa}$ 
sponding to the maximum transmittance was determined, whereby it was confirmed that the active damper was most effective at the resonance frequency (Fig. 10).

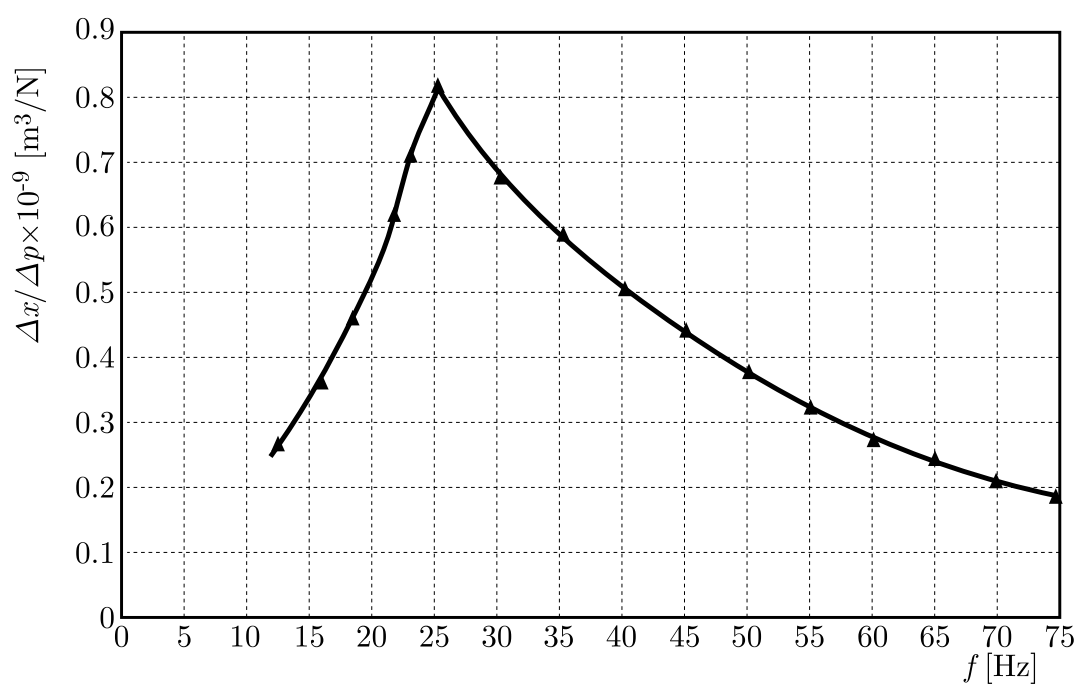

Fig. 10. Amplitude-frequency characteristic of the low-frequency damper at a forcing pressure of $12 \mathrm{MPa}$

\section{Conclusion}

A wide spectrum of pressure fluctuations occurs in hydraulic systems. Particularly hazardous and difficult to eliminate are pressure fluctuations in the range up to $50 \mathrm{~Hz}$, resulting in generation of infrasounds by machines equipped with a hydrostatic drive system.

Various hydropneumatic accumulators are commonly used to reduce pressure fluctuation amplitudes in the low-frequency range. The drawback of this solution is that hydropneumatic accumulators are most effective in reducing pressure fluctuation amplitudes when the accumulator resonance frequency coincides with the fluctuation frequency which is to be reduced. It should be noted that the accumulator eigenfrequency to a large extent depends on the magnitude of pressure in the hydraulic system incorporating this component. This means that hydropneumatic accumulators act selectively and so, as dampers, they are narrow-band filters, and their actual effectiveness in reducing pressure fluctuation is limited to practically a single excitation frequency equal to the hydropneumatic accumulator resonance frequency. Damping effectiveness has been observed to rapidly decrease already at slight deviations from the resonance frequency.

A concept of a pressure fluctuation damper whose key component is a hydropneumatic spring, effective in the range of low frequencies, has been presented in this paper. The axial cross section of the low-frequency damper, whose characteristic feature is that two hydropneumatic accumulators differing in their volume and gas precharge pressure form the hydropneumatic spring, is shown in Fig. 1. Owing to this solution, the hydropneumatic spring has an approximately constant stiffness in the whole load range, whereby its resonance frequency remains unchanged. Also, a mathematical model of the damper has been provided and effectiveness of the latter has been verified using a specially designed experimental setup enabling generation and recording of harmonic pressure fluctuations in a wide range of excitation frequencies. Theoretical analysis and verification results show that the low-frequency damper is most effective when its resonance frequency coincides with the frequency to be reduced. The damper resonance frequency, expressed by relation (3.12), depends on the equivalent capacitance of at least two hydroaccumulators (under heavier drive system loads), experimentally determined using defining relation (3.7) and on the hydraulic inductance described by relation (3.19). 
In hydrostatic drive systems, the low-frequency damper performs function of an acoustic filter, especially in low frequencies. This is evidenced by the chart in Fig. 11, showing a comparison between the third octave spectra of the sound pressure level $L_{m}$ of the actual hydraulic system lifting the jib of loader $\mathrm{E}-200$, with and without the low-frequency damper. The measurements have been carried out in a sound chamber in which the E-200 loader jib lifting system was placed. The chamber and the way in which the measurements were performed were described in detail in Kudźma (2012). The resonance frequency of the damper installed in the lifting system amounted to $25 \mathrm{~Hz}$, which corresponded to the pump driving motor rotational speed $n_{p}=1500 \mathrm{rpm}$. In order to reduce the pressure fluctuation amplitude for higher frequencies, one should install the passive damper described in, e.g., Kudźma and Kudźma (2015).

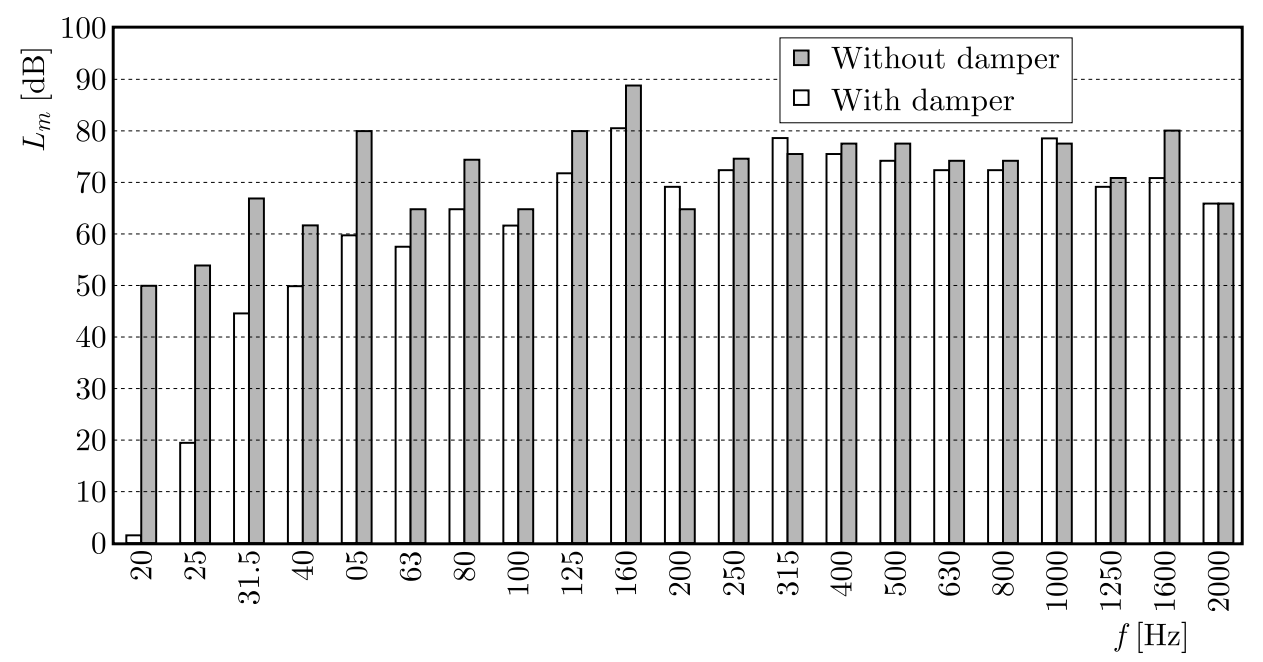

Fig. 11. Comparison between the third octave spectra of the sound pressure level $L_{m}$ of the actual Ł-200 loader jib lifting hydraulic system with and without the low-frequency damper, pump shaft rotational speed $n_{p}=1500 \mathrm{rpm}$, forcing pressure $p_{t}=12 \mathrm{MPa}$

\section{References}

1. BaCKÉ W., Murrenhoff H., 1994, Grundlagen der Ölhydraulik, Lecture notes: Institut für Fluidtechnische Antriebe und Steuerungen, RWTH Aachen, Germany

2. Changbin G., Zongxia J., 2014, A piezoelectric direct-drive servo valve with a novel multibody contacting spool-driving mechanism. Design, modelling and experiment, Proceedings of the Institution of Mechanical Engineers, Part C: Journal of Mechanical Engineering Science, 228, 1, $169-185$

3. Czerwinski A., Luczko J., 2015, Parametric vibrations of flexible hoses excited by a pulsating fluid flow, Part II: Experimental research, Journal of Fluids and Structures, 55, 174-190

4. Dindorf R., 2004, Modelling and Simulation of Nonlinear Control Elements and Systems of Fluid Drives (in Polish), Kielce University of Technology Publishing House, Kielce

5. Earnhart N.E., Cunefare K.A., 2012, Compact Helmholtz resonators for hydraulic systems, International Journal of Fluid Power, 13, 41-50

6. Garbacik A., Lisowski E., Szewczyk K., 1986, Hydraulic accumulator as pressure fluctuation damper (in Polish), Sterowanie i Napęd Hydrauliczny, 4, 9-13

7. German D.G., Reese J.M., Zhang Y.L., 2000, Vibration of a flexible pipe conveying viscous pulsating fluid flow, Journal of Sound and Vibration, 230, 2, 379-392

8. IJAs M., 2007, Damping of Low Frequency Pressure Oscillation, Tampere University of Technology Publication 656, Tampere 
9. Kollek W., Kudźma Z., Osiński P., Stosiak M., 2009, Low-frequency noise of heavy engineering machinery (in Polish), Napędy i Sterowanie, 1, 50-55

10. Kollek W., Kudźma Z., Rutański J., Stosiak M., 2010, Acoustic problems relating to microhydraulic components and systems, The Archive of Mechanical Engineering, 57, 3, 293-308

11. Kolvenbach H., Krips W., 2004, Revolution in Dynamik und Kraft: Neue Antriebs technologie für Stetigventile, 4th International Fluid Power Conference "Intelligent Solutions by Fluid Power", Dresden

12. Kudźma Z., 2012, Pressure Fluctuation and Noise Damping in Hydraulic Systems in Transient and Steady States (in Polish), Wrocław University of Technology Publishing House, Wrocław

13. Kudźma S., Kudźma Z., 2015, Refined model of passive branch damper of pressure fluctuations, Journal of Theoretical and Applied Mechanics, 53, 3, 557-567

14. Kudźma Z., Stosiak M., Herok S., 2014, Setup for determining static and dynamic characteristics of proportional valves (in Polish), Pomiary Automatyka Robotyka, 18, 3, 112-119

15. MichaŁowski S., Stolarski B., 1998, Suppression of Vibration and Noise in Heavy Engineering Machinery (in Polish), Monograph, Cracow University of Technology Publishing House, Cracow

16. Мikota J., 2000, Comparison of various designs of solid body compensators for the filtering of fluid flow pulsations in hydraulic systems, Proceedings of 1 FPNI-PhD Symposium, Hamburg

17. Ortwig H., Goebels K., Schwarz T., 1999, Hydroämpfer zur Geräuschreduzierung in hydraulischen Anlagen, Ölhydraulik und Pneumatik, 9, 652-656

18. Osiński P., Kollek W., 2013, Assessment of energetistic measuring techniques and their application to diagnosis of acoustic condition of hydraulic machinery and equipment, Archives of Civil and Mechanical Engineering, 13, 3, 313-321

19. Palczak E., Pomowski J., 2006, Transient states of hydraulic system with accumulator (in Polish), Inżynieria Maszyn, Rozwój Maszyn i Urzadzeń Hydraulicznych, 2-3, 29-38 